\title{
Direct observation of solute interstitials and their clusters in $\mathrm{Mg}$ alloys
}

\author{
Z.Q. Yang ${ }^{\mathrm{a}, \mathrm{b}, *}$, S.Y. Ma ${ }^{\mathrm{a}}$, Q.M. Hu ${ }^{\mathrm{a}}$, H.Q. Ye ${ }^{\mathrm{a}}$, M.F. Chisholm ${ }^{\mathrm{b}}$ \\ a Shenyang National Laboratory for Materials Science, Institute of Metal Research, Chinese Academy of Sciences, Shenyang 110016, China \\ b Materials Science and Technology Division, Oak Ridge National Laboratory, Oak Ridge, TN 37831, USA
}

\section{A R T I C L E I N F O}

\section{Keywords:}

Interstitial

Crowdion

Dumbbell

Aberration-corrected electron microscopy

Density functional theory

\begin{abstract}
A B S T R A C T
Individual solute interstitial atoms in Mg alloys were directly observed through aberration-corrected Z-contrast imaging. The atomic species and local positions of solute interstitials were further determined in combination of density functional theory calculations based on their formation energy. Individual octahedral and tetrahedral interstitials in hexagonal-close-packed $\mathrm{Mg}$ are $\mathrm{Zn}$ and $\mathrm{Y}$, respectively. Interestingly, there are six positions around the center of each octahedral interstice, which can also accommodate a Zn interstitial, besides the ideal octahedral interstice. Y tetrahedral interstitials evolve to [0001] Y-Mg dumbbells. Moreover, an Y atom can form a crowdion between two neighboring $\mathrm{Mg}$ atoms along the $<20 \overline{2} 3>$ direction. In particular, small clusters consisting of various point defects were observed, providing direct evidence for enthalpy-driven clustering of point defects, which may have implications for atomic-scale understanding of nucleation mechanisms of strengthening precipitates.
\end{abstract}

\section{Introduction}

Interstitials have important influences on various properties of materials [1-6]. Octahedral and tetrahedral interstitials are the mostly studied ones in metals and alloys so far [1,4,6-8]. A crowdion is a kind of interstitial with an atom inserted between two neighboring matrix atoms along a close-packed direction, instead of at the center of an interstice; and its center of mass can easily move along that axis, according to simulations [9]. Impurity interstitials can act as obstacles for dislocation motion; when they form atmospheres along dislocations, serrated flow, dynamic strain aging may occur during plastic deformation of metallic materials [10-12]. Large atoms prefer to substitute for matrix metal atoms, according to the Hume-Rothery rule. However, some large impurity atoms could diffuse even faster than small nonmetal atoms in hexagonal close packed (HCP) metals, such as Fe, Co and $\mathrm{Ni}$ in $\mathrm{Ti}$ and $\mathrm{Zr}$ [4]. The anomalously fast mobility of relatively large impurity metal atoms was attributed to an interstitial-mediated diffusion mechanism $[1,4]$, which may have potential applications on synthesis and processing of materials. The addition of a thin Ni foil between two Ti plates to be joined was able to greatly improve the weld quality [13]. Nucleation and growth of precipitates in metal matrix usually involves relocation of atoms from their original lattice sites to interstices, such as precipitates of Laves phase $\mathrm{MgZn}_{2}$ and icosahedral quasicrystal $\mathrm{Zn}_{6} \mathrm{Mg}_{3} \mathrm{Y}$ in $\mathrm{Mg}$ alloys [14].

It is thus important to study interstitials, in order to understand mechanisms underlying materials performances, such as strengthening, diffusion and solid-state phase transformations, which are closely associated with interstitials. Numerous theoretical modeling and computer simulations have been carried out about the structure and property of interstitials $[1,8,15]$. But, direct visualization of individual interstitials can provide useful knowledge about local atomic structures of the defects in materials, which is very helpful for understanding their dynamics and function [16-18]. Aberration-corrected scanning-transmission electron microscopy (STEM) is able to image single heavy dopant atoms with an atomic number, $\mathrm{Z}$, larger enough than the matrix atom, whether they were at the surfaces or embedded in the bulk lattice [19]. For example, substitutional atoms of Sb in Si were observed by high-angle annular dark field (HAADF) STEM imaging [20]. But, direct observation and identification of interstitials and crowdions in multicomponent alloys are still challenging.

$\mathrm{Zn}$ is an important alloying element in many Mg alloys [14,21-26]. And addition of rare-earth elements in $\mathrm{Mg}$ alloys may improve their ductility and creep resistance $[27,28]$. In this work, we studied $\mathrm{Zn}$ and $\mathrm{Y}$ interstitials in $\mathrm{Mg}$ alloys through combining aberration-corrected HAADF-STEM experimental observations and density functional theory (DFT). Besides conventional interstitial structures, including octahedral interstitials of $\mathrm{Zn}$ and Y-Mg [0001] dumbbells, Zn interstitials can also occupy positions slightly deviating from the ideal octahedral interstices, while individual $\mathrm{Y}$ atoms can exist as crowdions. Importantly, local clustering of interstitials was observed. Direct observation and identification of solute interstitials and their clusters may provide useful information for in-depth understanding and modeling a variety of

\footnotetext{
* Corresponding author at: Shenyang National Laboratory for Materials Science, Institute of Metal Research, Chinese Academy of Sciences, Shenyang 110016, China.

E-mail address: yangzq@imr.ac.cn (Z.Q. Yang).
} 
phenomena closely associated with point defects, such as diffusion, solute segregation and nucleation of precipitates, as well as their influences on the materials' performances.

\section{Materials and Method}

Binary Mg-2Zn and ternary Mg-1Zn-2Y (atomic \%) alloys were prepared by high frequency induction melting in a graphite crucible under argon atmosphere. Samples for electron microscopy observations were prepared by standard Ar ion milling. Samples were thinned to perforation by $3.5 \mathrm{keV}$ Ar ions, followed by surface cleaning at $0.5 \mathrm{keV}$. HAADF-STEM observations were performed on an aberration-corrected Nion UltraSTEM 100 microscope operated at $60 \mathrm{kV}$ which is below the threshold $(\sim 90 \mathrm{kV})$ for displacement damage in $\mathrm{Mg}[29,30]$. A beam convergence half-angle of $30 \mathrm{mrad}$ was used, which provided a focus depth of about $4 \mathrm{~nm}$ [31]. A detector half-angle range of 86-200 mrad was used to record HAADF-STEM images. Atomic resolution imaging was usually performed in regions with a sample thickness below $20 \mathrm{~nm}$, according to measurements of electron energy loss spectroscopy. Image simulations were performed using a package based on a multislice algorithm [32].

First-principles calculations were performed with density functional theory (DFT) as implemented in the Vienna ab initio Simulation Package [33]. The electron exchange and correlation were described with the generalized gradient approximation of Perdew, Burke and Ernzerhof [34] and the electron-ion interaction was represented by the projector augmented wave method [35], with plane wave up to an energy cutoff of $350 \mathrm{eV}$. The formation energy of a model containing $m$ $\mathrm{Mg}$ atoms and $l, n$ interstitial $\mathrm{Zn}, \mathrm{Y}$ atoms was be calculated by

$\Delta E_{f}=E\left(\mathrm{Mg}_{m} \mathrm{Zn}_{l} \mathrm{Y}_{n}\right)-m E(\mathrm{Mg})-l E(\mathrm{Zn})-n E(\mathrm{Y})$,

where $E\left(\mathrm{Mg}_{m} \mathrm{Zn}_{l} \mathrm{Y}_{n}\right)$ is the total energy of interested defect configurations, and $E(X, X=\mathrm{Mg}, \mathrm{Zn}, \mathrm{Y})$ is the energy of per $X$ atom in its bulk. To eliminate the unusual size dependence of formation energy calculation, large supercells containing $5 \times 5 \times 4 \mathrm{Mg}$ unit cells were used, with the theoretical lattice constants of $a=3.209$ and $c=5.120 \AA$ for HCP Mg.

\section{Results and Discussion}

Fig. 1 shows positions for two types of widely discussed interstitials, an octahedral interstitial (OI) and a tetrahedral interstitial (TI), in HCP crystals schematically, which will be used to identify the type of interstitials observed by HAADF-STEM imaging. Besides OI and TI, there may be other six symmetrical interstitial configurations, such as [0001] dumbbell (DB), crowdion (C), basal split, basal crowdion, basal octahedral, basal tetrahedral [36].

First-principles calculations show that the formation energies of both $\mathrm{Zn}(-0.03 \mathrm{eV} /$ atom $)$ and $\mathrm{Y}(-0.09 \mathrm{eV} /$ atom $)$ substitutions are negative (Table 1). This is consistent with experimental observations of atomic columns showing enhanced brightness omnipresent in all $\mathrm{Mg}$ grains observed by HAADF-STEM (see one example shown by the profile overlapped on Fig. 2(a)). Interestingly, intensity spots at nonlattice sites were also observed in $\mathrm{Mg}$ grains of both alloys. Fig. 2 shows atomic resolution HAADF-STEM images recorded along the $\langle 1 \overline{2} 10\rangle \mathrm{Mg}$ zone axis for the Mg-Zn-Y alloy. There are extra intensity spots at nonlattice positions like these indicated by circles and squares in HCP Mg lattice, which was never observed in pure $\mathrm{Mg}$ samples (Supplementary Fig. S1). Interestingly, coexistence of several non-lattice intensity spots was observed more frequently (Fig. 2(c) and (d)), compared with single ones (Fig. 2(a)). The brightness of one Zn or Y atom is roughly 5 or 8 times that of a Mg atom in HAADF-STEM images [19]. These intensity spots indicated by circles and squares are attributed to OI and TI atoms (Fig. 1), respectively. The extra intensity spot indicated by an arrow in Fig. 2c implies presence of a solute crowdion out of the HCP basal plane. The intensity spots indicated by rhombuses in Fig. 2(d) are resulted from solute atoms at positions deviating slightly from tetra-
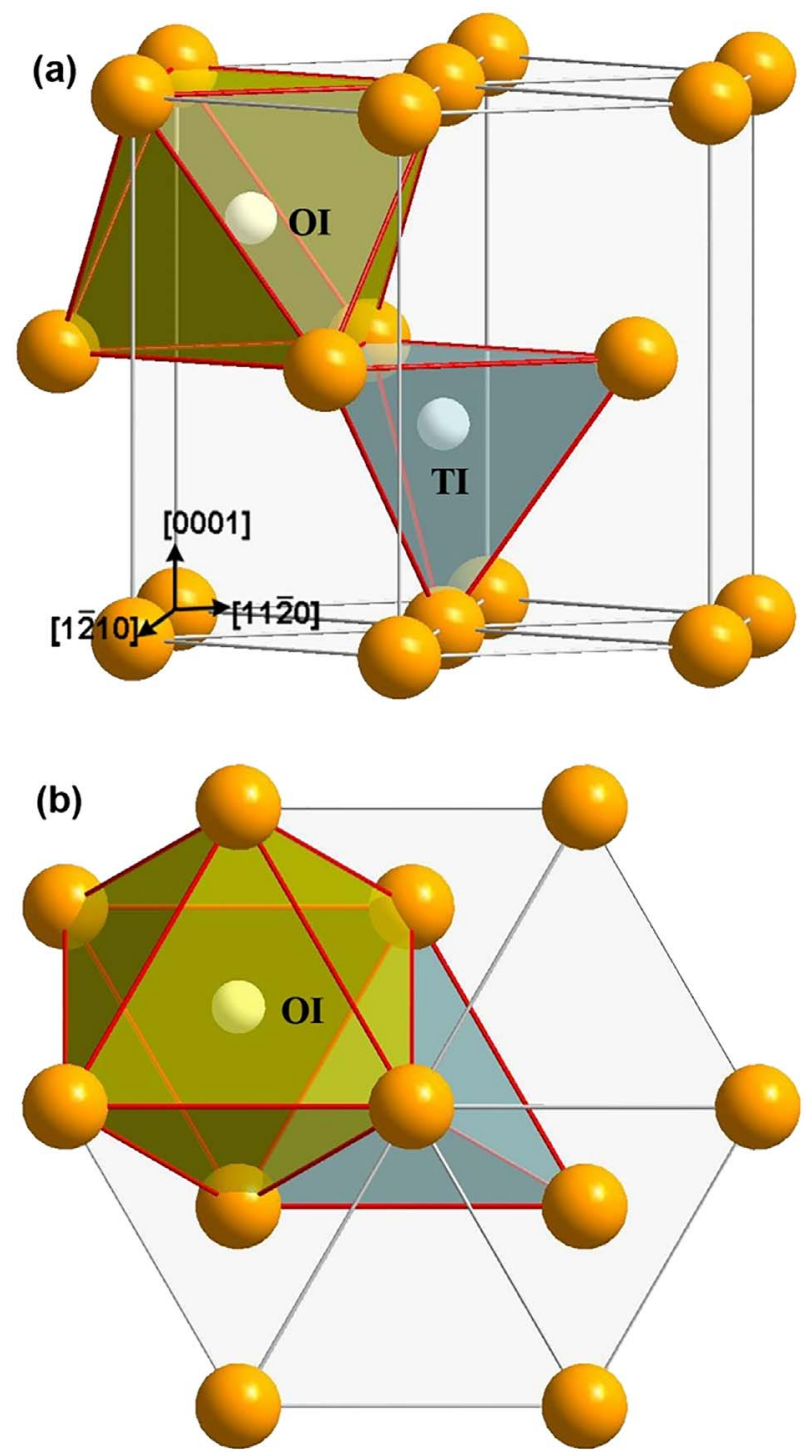

Fig. 1. Schematic models showing interstitials of OI and TI in HCP structures viewed along: (a) a direction close to [12 10$]$ and (b) [0001], respectively. The TI atom is along with a Mg column viewed along the [0001] zone axis.

Table 1

Calculated formation energies, $E_{f}$ (in $\mathrm{eV}$ ) of substitutionals (Sub), interstitials and crowdions in $\mathrm{Mg}$.

\begin{tabular}{lllllll}
\hline & Sub & OI & TI & C & OID & 3 Zn +1 Y \\
\hline $\mathrm{Zn}$ & -0.03 & 1.15 & 1.57 & 1.32 & 1.12 & $5.39^{\mathrm{a}}$ \\
$\mathrm{Y}$ & -0.09 & 3.27 & $2.87^{\mathrm{b}}$ & 2.97 & 3.18 & \\
$\mathrm{Mg}$ & - & 2.39 & $2.33^{\mathrm{b}}$ & 2.19 & - & \\
\hline
\end{tabular}

a $3 Z n$ OIDs + 1Y-Mg DB.

b Y-Mg or Mg-Mg DB.

hedral interstices. Additionally, the Mg column indicated by an arrow in Fig. 2(d) shows an intensity spot with an extended tail, suggesting presence of a solute atom at its immediate vicinity close to an octahedral interstice marked by the dashed circle. The observed interstitials and crowdions should not be produced by knock-on damage, since the high tension applied is below the threshold value for $\mathrm{Mg}$ [29]. Atomic species of these point defects cannot be readily and accurately determined directly from the STEM images, since the corresponding intensity depends not only on the atomic number of the solute atom, but also its depth in the sample [19]. 

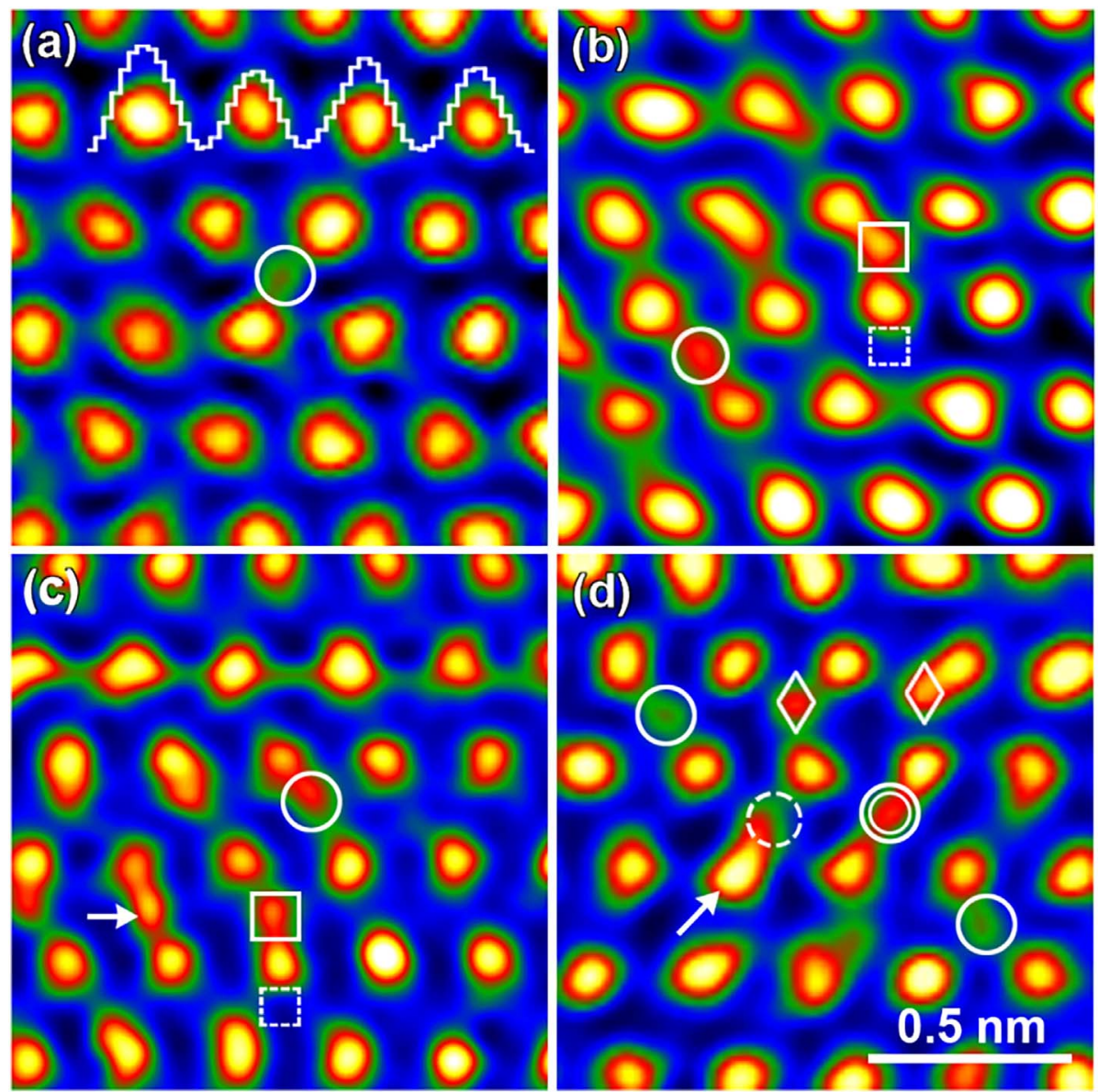

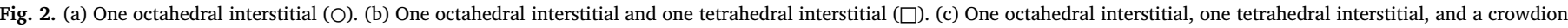

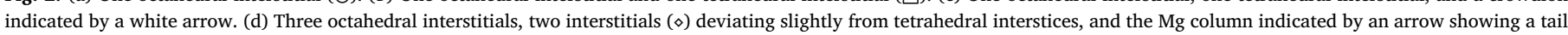

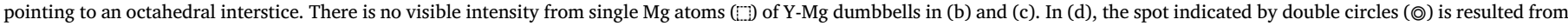

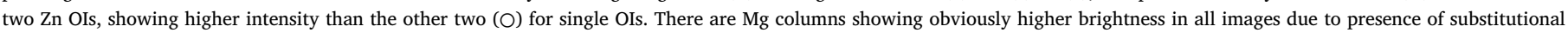
solute atoms, e.g., the leftmost peak of the intensity profile overlapped on (a).

First-principles calculations show that formation energies of an OI and a TI of $\mathrm{Zn}$ are respectively $1.15 \mathrm{eV}$ and $1.57 \mathrm{eV}$ (Table 1), implying that $\mathrm{Zn}$ would form OIs (Fig. 3(a) and (c)) instead of TIs. Y would form TIs which evolve into a [0001] Y-Mg DB finally (Fig. 3(e)), according to DFT calculations. Therefore, those extra spots indicated by circles and squares on HAADF-STEM images shown in Fig. 2 are attributed to the presence of octahedral Zn and tetrahedral Y interstitials, respectively. The single $\mathrm{Mg}$ atom of an Y-Mg [0001] dumbbells could not produce visible intensity on HAADF-STEM images (Figs. 2(b), (c) and 3(e)). The intensity spots from the $\mathrm{Y}$ atoms of the $\mathrm{Y}-\mathrm{Mg}$ DBs are usually higher than those from $\mathrm{Zn}$ OIs in the same region, since the atomic number of $\mathrm{Y}$ $(\mathrm{Z}=39)$ is higher than $\mathrm{Zn}(\mathrm{Z}=30)$. This is also confirmed by image simulations (Fig. 3(a) and (e)). Additionally, based on the calculated formation energies, it is deduced that the possibility for the presence of an $\mathrm{Y}$ crowdion is about 15 times that of a $\mathrm{Zn}$ crowdion in a Mg-Zn-Y solid solution, according to the Boltzmann equation of statistical thermodynamics [37]. Therefore, crowdions in the Mg-Zn-Y solid solution are likely scattered Y atoms (Figs. 2(c) and 3(f)). Presence of crowdions was only predicted theoretically in previous studies [9]. Our atomic resolution observations provided direct evidence for the formation of individual crowdions in HCP metals experimentally.

Individual $\mathrm{Y}$ tetrahedral interstitials in $\mathrm{Mg}$ will evolve into $\mathrm{Y}-\mathrm{Mg}$ dumbbells (Fig. 3(e)). However, it was observed that there were two TIs which were not in the DB configuration at a region with also several $\mathrm{Zn}$ interstitials below them, as indicated by rhombuses in Fig. 2(d). This configuration was confirmed by first-principles calculations (Fig. 3(g)). The presence of three $\mathrm{Zn}$ OIs in $\mathrm{Mg}$ makes it hard for the $\mathrm{Mg}$ atom on top of them to deviate from its HCP lattice site obviously (Fig. 3(g)). Therefore, an Y interstitial atom would stay at a position nearby a tetrahedral interstice, if several neighboring Zn OIs were present in HCP Mg lattice (Figs. 2(d) and 3(g)). At the same time, the strain field from the $\mathrm{Y}$ interstitial made the $\mathrm{Zn}$ interstitials deviating from the octahedral interstices to some extent. The left $\mathrm{Zn}$ interstitial shown in Fig. $3(\mathrm{~g})$ is close to the neighboring $\mathrm{Mg}$ atomic column, which might result in the appearance of an extended tail of the intensity spot for the Mg column (Fig. 2(d)).

Interestingly, first-principles calculations show that isolated $\mathrm{Zn}$ interstitials deviating slightly from octahedral interstices (OIDs) (Fig. 3(b) and (d)) have a formation energy $0.03 \mathrm{eV}$ lower than that of an ideal $\mathrm{Zn}$ OI in $\mathrm{Mg}$. There are six positions with a point group of $\overline{3}$ around the center of an octahedral interstice in Mg. The decreased formation energy of OIDs is thus associated with their higher multiplicity of 6 , compared with a multiplicity of 1 for OIs in HCP metals, according to theory of statistical thermodynamics [37]. This little difference in formation energies between OIs and OIDs of $\mathrm{Zn}$ implies similar concentrations for these two kinds of interstitials in Mg-Zn solid solution, considering the uncertainty of about $0.05 \mathrm{eV}$ of the calculated energies. A Zn OID is at a position quite close to one edge of a triangle connecting three neighboring $\mathrm{Mg}$ atoms in a basal plane viewed along [0001] (Fig. 3(d)). This theoretical prediction was confirmed by experimental observations, as shown in Fig. 4. There are respectively one and six intensity spots from OIDs in regions shown in Fig. 4(a) and (c), as indicated by triangles. Presence of interstitials with lowsymmetry configurations was also predicted by first-principles calcula- 


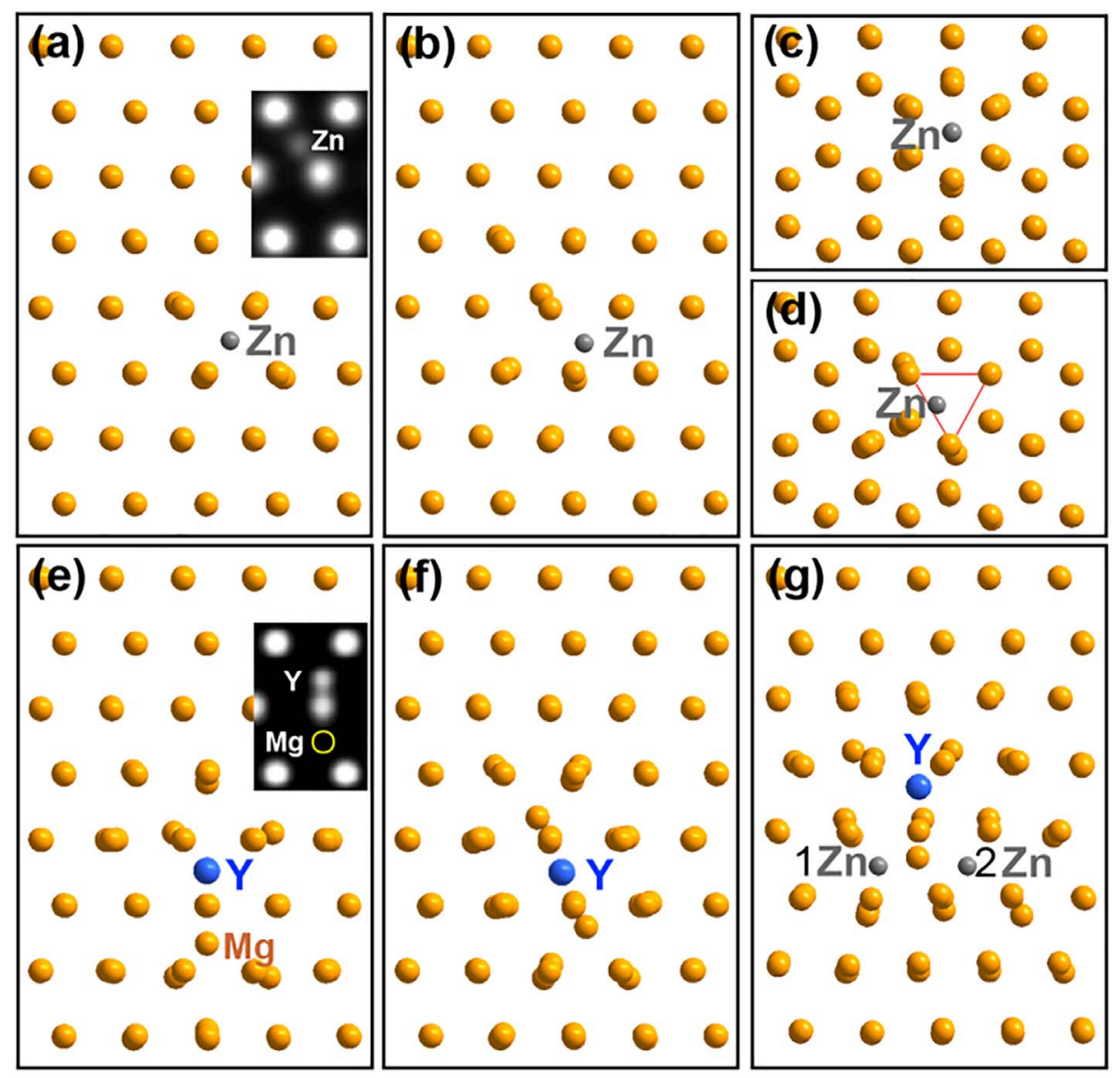

Fig. 3. (a) and (c) Zn OI viewed along $\langle 1 \overline{2} 10\rangle$ and [0001], respectively. (b) and (d) Zn OID viewed along $\langle 1 \overline{2} 10\rangle$ and [0001], respectively. (e) and (f) $\langle 1 \overline{2} 10\rangle$ projections of an Y-Mg [0001] DB and one $\mathrm{Y}$ crowdion, respectively. (g) Relaxed configuration for co-existence of three $\mathrm{Zn}$ OIDs and one $\mathrm{Y}$ TI $(3 \mathrm{Zn}+1 \mathrm{Y}$ ) in Mg. The Mg atom of the Y-Mg DB is originally at a lattice site shared by the three $\mathrm{Mg}$ octahedra holding the three $\mathrm{Zn}$ interstitials. Overlapped on defect models shown in (a) and (e) are simulated images for a Zn OI and an Y-Mg DB in samples of $5.2 \mathrm{~nm}$ thick, without consideration of local lattice distortion around the point defects.

tions in other HCP metals, such as Ti, $\mathrm{Zr}$ and Hf [8]. Those extra intensity spots indicated by circles on HAADF-STEM images (Fig. 4(a) and (c)) are thus associated with Zn OIs. Although first-principles calculations (Table S1) indicated that Zn might form basal octahedral interstitials, since the formation energy is quite close to that of normal OIs, basal octahedral interstitials have not been observed so far experimentally.

The formation energy for the complex defect structure consisting of three $\mathrm{Zn}$ interstitials and one Y-Mg DB (Fig. $3(\mathrm{~g})$ ) is $5.39 \mathrm{eV}$ which is almost $1.0 \mathrm{eV}$ lower than the sum $(6.32 \mathrm{eV})$ of formation energies of the four isolated defects. Therefore, interstitials and crowdions within a local region might segregate to form clusters, which is driven by decrease of local energy [38,39], as shown in Figs. 2 and 4. In fact, larger clusters with more interstitials were also observed experimentally in both alloys. Fig. 5(a) shows an atomic resolution HAADF-STEM image for a region with a high density of point defects including $\mathrm{Zn}$ OIDs, Y-Mg DBs and one Y crowdion in the Mg-Zn-Y alloy. Shown in Fig. 5(b) is a region containing high density of Zn OIDs in the Mg-Zn alloy. Furthermore, there are two or more Zn OIDs at different depths for positions indicated by black triangles in Fig. 5(b), according to their higher intensities compared with those indicated by red ones.

Individual interstitials and crowdions of large atoms are high energy defects (Table 1). Therefore, isolated $\mathrm{Zn}$ or Y interstitials were seldom observed in regions without substitutional and/or interstitial $\mathrm{Zn} / \mathrm{Y}$ atoms in the same local region (Fig. 2). But, our atomic resolution observations provided direct evidence for the enhanced stability of small clusters containing solute interstitials and crowdions in HCP Mg. First-principles calculations demonstrate that clustering of point defects is driven by decrease of enthalpies (Fig. 3 and Table 1). Our atomic- scale observations of tiny clusters of solute interstitials in Mg provide pertinent evidence for enhanced stability of clusters of point defects previously observed by molecular dynamics simulations and lowmagnification phase contrast observations $[15,39]$. Computer simulations found that interstitial atoms did not recombine with vacancies at the periphery of tiny dislocation loops formed by self-interstitials [15]. Interestingly, coarsening of interstitial dislocation loops in $\alpha$-Fe, instead of shrinkage, was observed through phase-contrast imaging in a transmission electron microscope during in situ heating experiments [39].

In addition, compositional fluctuations are usually omnipresent in real alloys. There are always atomic columns with $\mathrm{Zn}$ or $\mathrm{Y}$ substitutional atoms at positions where solute interstitials were observed experimentally (Figs. 2, 4, 5, and see Fig. S2 for a much larger region), implying that the formation energies for solute interstitials within local regions enriched in $\mathrm{Zn}$ and/or Y would be decreased to some extent below the calculated ones (Table 1), which benefited the formation of solute interstitials. Local structural transformations would occur through reorganization of atoms, if the chemical composition within a region reached a level sufficient for stabilizing a structure different from the matrix [40]. The observed small clusters containing solute interstitials/crowdions with enhanced stability might be embryos which could trigger formation of strengthening precipitates in $\mathrm{Mg}$ grains.

\section{Conclusion}

In summary, we have observed Zn and Y interstitials in HCP Mg by aberration-corrected HAADF-STEM imaging. Zn atoms may form OIs or 

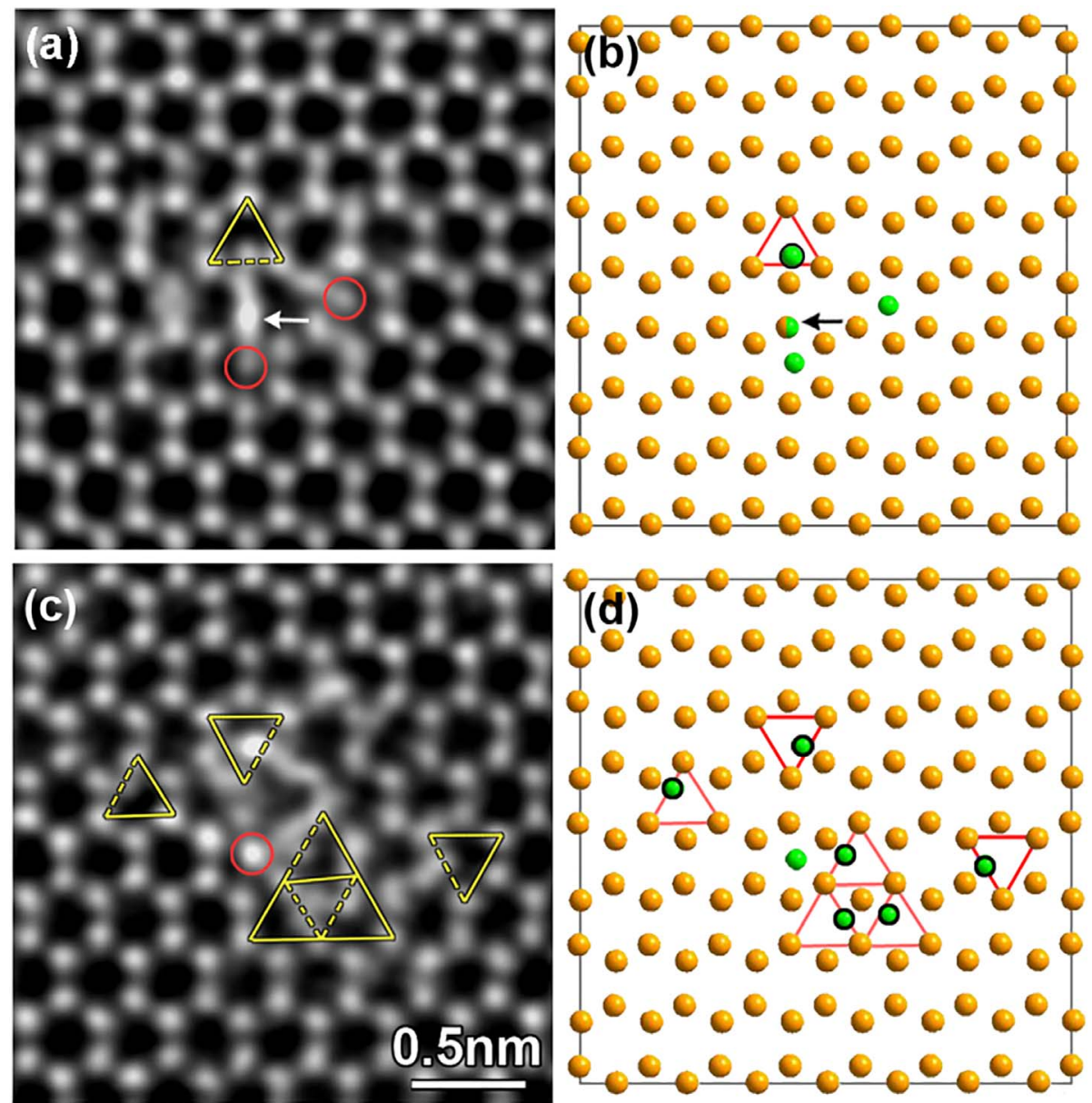

$\mathrm{Zn}: \cdot \mathrm{Ol} O \mathrm{OID}$

- substitution

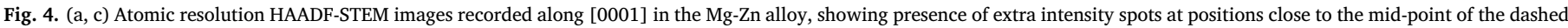

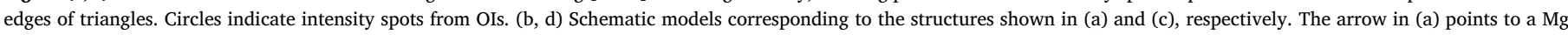
column with Zn substitutional atom(s). No lattice distortion is taken into account in the schematic models for simplicity.

OIDs in $\mathrm{Mg}$, while $\mathrm{Y}$ atoms may exist as $\mathrm{Y}-\mathrm{Mg}$ dumbbells or crowdions out of basal planes. The formation of Zn OIDs and Y crowdions was demonstrated experimentally with atomic resolution for the first time. More importantly, solute interstitials or crowdions were often found in configurations of small clusters, in good agreement with the reduced formation energies from DFT calculations. Our investigations show how powerful it is to combine atomic resolution $Z$-sensitive microscopy imaging with DFT calculations in studying point defects and their tiny
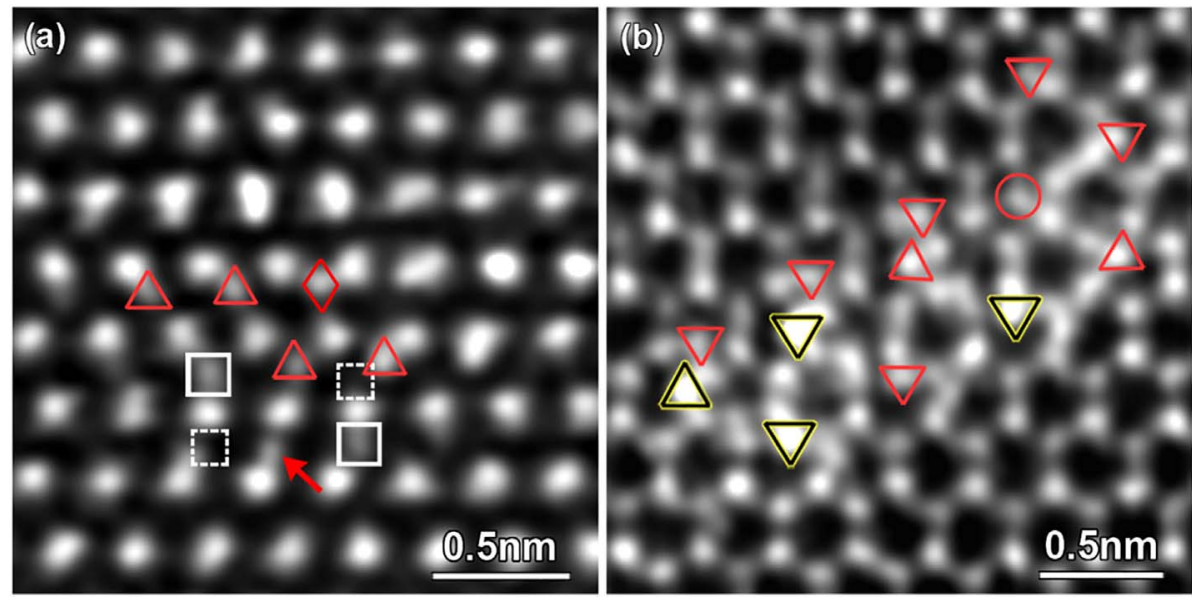

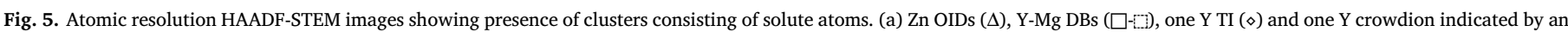

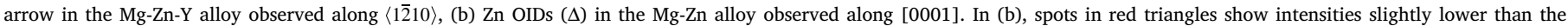

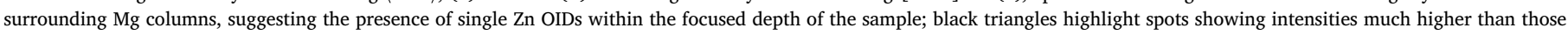

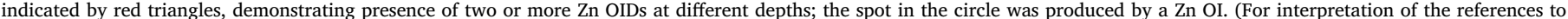
colour in this figure legend, the reader is referred to the web version of this article.) 
clusters in multicomponent materials, which may provide important information for understanding many solid-state processes on the atomic level.

\section{Acknowledgements}

This research was supported by the National Natural Science Foundation of China (51371178, 51390473) and Liaoning Province (2015020237). Work at ORNL (Z.Q.Y. and M.F.C.) was supported by the Department of Energy, Office of Science, Basic Energy Sciences, Materials Sciences and Engineering Division. Atomic resolution imaging was conducted at the Center for Nanophase Materials Sciences, which is supported by DOE Office of Science User Facility.

\section{References}

[1] N. Bernstein, A. Shabaev, S.G. Lambrakos, First principles study of normal and fast diffusing metallic impurities in hcp titanium, Comput. Mater. Sci. 109 (2015) 380-387.

[2] J.M.G. Jimenez, J. Cembrero, M. Mollar, B. Mari, Photoluminescent properties of electrochemically synthetized ZnO nanotubes, Mater. Charact. 119 (2016) $152-158$.

[3] H. Bracht, S. Schneider, J.N. Klug C.Y. Liao, J.L. Hansen, E.E. Haller, A.N. Larsen, D. Bougeard, M. Posselt, C. Wundisch, Interstitial-mediated diffusion in germanium under proton irradiation, Phys. Rev. Lett. 103 (2009) 4.

[4] G.M. Hood, R.J. Schultz, Ultra-fast solute diffusion in alpha-Ti and alpha-Zr, Philos. Mag. 26 (1972) 329-336.

[5] V.P. Markevich, S. Leonard, A.R. Peaker, B. Hamilton, A.G. Marinopoulos, J. Coutinho, Titanium in silicon: lattice positions and electronic properties, Appl. Phys. Lett. 104 (2014) 4.

[6] R.C. Pasianot, R.A. Perez, First-principles appraisal of solute ultra-fast diffusion in hcp Zr and Ti, J. Nucl. Mater. 434 (2013) 158-161.

[7] W. Schilling, Self-interstitial atoms in metals, J. Nucl. Mater. 69-7 (1978) 465-489.

[8] G. Verite, C. Domain, C.C. Fu, P. Gasca, A. Legris, F. Willaime, Self-interstitial defects in hexagonal close packed metals revisited: evidence for low-symmetry configurations in Ti, Zr, and Hf, Phys. Rev. B 87 (2013) 134108.

[9] D.A. Terentyev, L. Malerba, M. Hou, Dimensionality of interstitial cluster motion in bcc-Fe, Phys. Rev. B 75 (2007) 104108.

[10] A.H. Cottrell, B.A. Bilby, Dislocation theory of yielding and strain ageing of iron, Proc. Phys. Soc. Lond. A 62 (1949) 49-62.

[11] J.P. Hirth, J. Lothe, Theory of Dislocations, second ed., Wiley, New York, 1982.

[12] J. Wilde, A. Cerezo, G.D.W. Smith, Three-dimensional atomic-scale mapping of a Cottrell atmosphere around a dislocation in iron, Scr. Mater. 43 (2000) 39-48.

[13] R.K. Everett, J.N. Wolk, A. Shabaev, S.G. Lambrakos, Issues Concerning Small Additions of Ni to Ti Friction Stir Welds, (2013).

[14] J.F. Liu, Z.Q. Yang, H.Q. Ye, Direct observation of solid-state reversed transformation from crystals to quasicrystals in a Mg alloy, Sci. Rep.-Uk 5 (2015) 09816.

[15] M.A. Puigvi, N. De Diego, A. Serra, Y.N. Osetsky, D.J. Bacon, On the interaction between a vacancy and self-interstitial atom clusters in metals, Philos. Mag. 87 (2007) 3501-3517.
[16] J.F. Nie, Y.M. Zhu, J.Z. Liu, X.Y. Fang, Periodic segregation of solute atoms in fully coherent twin boundaries, Science 340 (2013) 957-960.

[17] S.J. Pennycook, W. Zhou, S.T. Pantelides, Watching atoms work: nanocluster structure and dynamics, ACS Nano 9 (2015) 9437-9440.

[18] Z.Q. Yang, L.C. Yin, J. Lee, W.C. Ren, H.M. Cheng, H.Q. Ye, S.T. Pantelides, S.J. Pennycook, M.F. Chisholm, Direct observation of atomic dynamics and silicon doping at a topological defect in graphene, Angew. Chem. Int. Ed. 53 (2014) 8908-8912.

[19] S.J. Pennycook, Z-contrast transmission electron-microscopy - direct atomic imaging of materials, Annu. Rev. Mater. Sci. 22 (1992) 171-195.

[20] P.M. Voyles, D.A. Muller, J.L. Grazul, P.H. Citrin, H.J.L. Gossmann, Atomic-scale imaging of individual dopant atoms and clusters in highly $n$-type bulk Si, Nature 416 (2002) 826-829.

[21] J. Hiscocks, B.J. Diak, A.P. Gerlich, M.R. Daymond, Formation mechanisms of periodic longitudinal microstructure and texture patterns in friction stir welded magnesium AZ80, Mater. Charact. 122 (2016) 22-29.

[22] J. Jiang, M. Song, H. Yan, C. Yang, S. Ni, Deformation induced dynamic recrystallization and precipitation strengthening in an Mg-Zn-Mn alloy processed by high strain rate rolling, Mater. Charact. 121 (2016) 135-138.

[23] B. Langelier, A. Korinek, P. Donnadieu, S. Esmaeili, Improving precipitation hardening behavior of $\mathrm{Mg}$-Zn based alloys with Ce-Ca microalloying additions, Mater. Charact. 120 (2016) 18-29.

[24] W.W. Hu, Z.Q. Yang, H.Q. Ye, Cottrell atmospheres along dislocations in longperiod stacking ordered phases in a Mg-Zn-Y alloy, Scr. Mater. 117 (2016) 77-80.

[25] S. Amira, D. Dube, R. Tremblay, E. Ghali, Microstructure of die cast and thixocast ZAEX10430 (Mg-10Zn-4Al-3Ce-0.3Ca) alloy, Mater. Charact. 76 (2013) 48-54.

[26] Z. Li, J.X. Zheng, B. Chen, Unravelling the structure of gamma in Mg-Gd-Zn: an atomic-scale HAADF-STEM investigation, Mater. Charact. 120 (2016) 345-348.

[27] M.E. Drits, Z.A. Sviderskaya, L.L. Rokhlin, N.I. Nikitina, Effect of alloying on the properties of Mg-Gd alloys, Met. Sci. Heat Treat. 21 (1979) 887-889.

[28] Y. Jie, W. Qudong, Y. Dongdi, W. Huan, C. Changjiang, Y. Bing, Creep behavior of Mg-9Gd-1Y-0.5Zr (wt.\%) alloy piston by squeeze casting, Mater. Charact. 78 (2013) $37-46$.

[29] L.W. Hobbs, Radiation effects in analysis by TEM, in: J.J. Hren, J.I. Goldstein, D.C. Joy (Eds.), Introduction to Analytical Electron Microscopy, Plenum Press, New York, 1987, pp. 399-445.

[30] O.L. Krivanek, N. Dellby, M.F. Murfitt, M.F. Chisholm, T.J. Pennycook, K. Suenaga, V. Nicolosi, Gentle STEM: ADF imaging and EELS at low primary energies, Ultramicroscopy 110 (2010) 935-945.

[31] A.R. Lupini, A.Y. Borisevich, J.C. Idrobo, H.M. Christen, M. Biegalski, S.J. Pennycook, Characterizing the two- and three-dimensional resolution of an improved aberration-corrected STEM, Microsc. Microanal. 15 (2009) 441-453.

[32] C.T. Koch, Determination of Core Structure Periodicity and Point Defect Density Along Dislocations, Arizona State University, 2002.

[33] G. Kresse, J. Furthmuller, Efficient iterative schemes for ab initio total-energy calculations using a plane-wave basis set, Phys. Rev. B 54 (1996) 11169-11186.

[34] J.P. Perdew, K. Burke, M. Ernzerhof, Generalized gradient approximation made simple, Phys. Rev. Lett. 77 (1996) 3865-3868.

[35] G. Kresse, D. Joubert, From ultrasoft pseudopotentials to the projector augmentedwave method, Phys. Rev. B 59 (1999) 1758-1775.

[36] Q. Peng, W. Ji, H. Huang, S. De, Axial ratio dependence of the stability of selfinterstitials in HCP structures, J. Nucl. Mater. 437 (2013) 293-296.

[37] A.H. Carter, Classical and Statistical Thermodynamics, Prentice-Hall, New Jersey, 2001.

[38] N.E.B. Cowern, A. Cacciato, J.S. Custer, F.W. Saris, W. Vandervorst, Role of C and B clusters in transient diffusion of B in silicon, Appl. Phys. Lett. 68 (1996) 1150-1152.

[39] S. Moll, T. Jourdan, H. Lefaix-Jeuland, Direct observation of interstitial dislocation loop coarsening in alpha-iron, Phys. Rev. Lett. 111 (2013) 015503.

[40] T.D. De la Rubia, G. Gilmer, Cluster nucleation - watching nanoclusters nucleate, Nat. Mater. 1 (2002) 89-90. 